\title{
Infecção pelos vírus das hepatites B e D entre grupos indígenas da Amazônia Brasileira: aspectos epidemiológicos
}

\author{
Hepatitis B and D virus infection within amerindians ethnic groups \\ in the Brazilian Amazon: epidemiological aspects
}

Wornei Silva Miranda Braga ${ }^{1}$

\begin{abstract}
RESUM0
Entre populações autóctones da América, estudos relatam altos índices de infecção e doença pelos vírus das hepatites B e D. Esta é uma revisão do que já foi descrito entre indígenas da Amazônia brasileira. Em alguns grupos a prevalência do AgHBs é muito baixa, enquanto que outros da mesma região, apresentam padrão de elevada endemicidade, presente inclusive entre menores de 10 anos. OVHD só foi encontrado entre etnias no estado do Amazonas. É descrito a importância da transmissão horizontal familiar, e do contato sexual entre adultos jovens. Fatores socioculturais, genéticos, ecológicos, e a formação histórica desses povos, são apontados como determinantes deste padrão. Entretanto, a origem do VHB e VHD na Amazônia é ainda obscura. Populações indígenas com sua memória genética são, na verdade, 0 experimento ao vivo, o que demanda investigação abrangente, avaliando a influência dos aspectos históricos, ecológicos, médicos e antropológicos envolvidos, utilizando inclusive técnicas modernas de biologia molecular.
\end{abstract}

Palavras-chaves: Hepatite B. Hepatite delta. Indígenas. Amazônia.

\begin{abstract}
Several studies describe very high prevalence rates of infection and disease of hepatitis B and D within Native American population. This is a review of what has been described among Amerindians of Brazilian Amazon. Some groups show low prevalence rates of $\mathrm{HBsAg}$, whereas, others of the same region reveal high endemic pattern, even among individuals less then 10 years of age. $\mathrm{HDV}$ is only found in groups of Amazonas province. Transmission may occur by interfamilial dissemination or sexual contact among young adults. Socio-cultural, genetic, and ecological factors are described as determinants of this unique pattern. Nevertheless, the origin of these two viruses is yet to be disclosed. Amerindians population and their genetic memory are a live experiment, which demands a broad investigation, weighting with modern tools, as molecular biology, the influence of historical, genetic, medical and anthropological factors.
\end{abstract}

Key-words: Hepatitis B. Hepatitis delta. Prevalence. Amerindians.

A infecção pelo vírus da hepatite B (VHB) talvez seja a virose mais comum do gênero humano. A grande maioria dos casos, concentra-se no Sudeste da Ásia, Amazônia, África, Pacífico sul e extremo norte da América. Estima-se que estas regiões de elevada endemicidade contribuam com mais de $90 \%$ dos casos mundiais, sendo o VHB implicado como importante fator na etiologia de hepatites crônica, cirrose hepática e hepatocarcinoma ${ }^{30} 40$.

0 vírus da hepatite $\mathrm{D}$ (VHD) está diretamente relacionado ao VHB, pois sendo um vírus defectivo necessita da presença deste para infectar um indivíduo susceptível. Em certas regiões de elevada endemicidade do VHB, está presente entre $25 \%$ a
$30 \%$ dos portadores do AgHBs 13711193134 e é freqüentemente associado a surtos de hepatite fulminante e evolução precoce para formas graves de hepatopatia crônica ${ }^{10} 12182239$.

Entre populações autóctones da América, estudos relatam altas taxas de prevalência de infecção e doença pelo VHB e VHD na Amazônia venezuelana ${ }^{22} 323738$, colombiana ${ }^{10}$, peruana ${ }^{11}{ }^{16}$, equatoriana ${ }^{29}$, boliviana ${ }^{26}$ e brasileira ${ }^{268131517203738}$.

Este é um artigo de revisão e atualização de informações. Publicações dos últimos quinze anossobre e epidemiologia da infecção pelos VHB e VHD na região Amazônica, são nosso objeto de estudo.

As informações são analisadas em busca de fatos comuns e peculiaridades. Os seguintes indicadores são definidos

\footnotetext{
1. Fundação de Medicina Tropical do Amazonas, Manaus, AM.

Endereço para correspondência: Dr. Wornei Braga. Fundação de Medicina Tropical do Amazonas. Av. Pedro Teixeira 25, D. Pedro I, 69040-000 Manaus, AM. Fax: 92 238-3762.

e-mail:wornei@ hotmail.com
} 
como pontos chaves para a elaboração deste texto: prevalência de marcadores virais e prováveis mecanismos de manutenção da transmissão. As informações apresentadas são referentes a estudos realizados em comunidades indígenas da Amazônia brasileira, e estudos em outras regiões e entre populações não indígenas, são utilizados como parâmetros de avaliação dos resultados e indicativos de novas abordagens.

\section{PREVALÊNCIA DE MARCADORES VIRAIS}

A prevalência de portadores crônicos do AgHBs relatada, revela padrão heterogêneo, com etnias apresentando taxas muito baixas e outros grupos da mesma região com padrão de elevada endemicidade, como por exemplo, $0 \%$ entre os Jamamadi e $20,6 \%$ entre os Paumari, no Estado do Amazonas, 0,6\% entre os Mundurucú e 14,4\% entre os Parakanã no Pará, 1\% entre os Cinta Larga e 11,3\% entre os Suruí em Rondônia, e 1,9\% entre os Caiabi e 6,9\% entre os Txucarramãe no Mato Grosso (Tabela 1). Algumas dessas publicações também demonstram variação estatisticamente significativa na distribuição deste marcador entre aldeias de um mesmo grupo étnico ${ }^{83}$.

Em relação à presença do AgHBs por grupo de idade, nas etnias em que a prevalência deste marcador apresenta um padrão de moderada ou elevada endemicidade, observa-se a presença significativa deste marcador desde pequenas idades, como entre os Paumari, no Amazonas (Tabela 2). Não é relatado reatividade para 0 anti- $\mathrm{HBC}$ IgM, e a presença do AgHBe só é descrita em menores de 10 anos de idade, aglomerados em núcleos familiares ${ }^{8}$.

A taxa de prevalência de infecção passada, indivíduos anti$\mathrm{HBc}$ total reativo, alcança índices de mais de $95 \%$ entre os

Tabela 1 - Prevalência de marcadores do VHB e VHD entre indígenas da Amazônia Brasileira, por etnia e estado.

$\begin{array}{lcrrrr}\text { Etnia } & \text { Estado } & \text { AgHBs } & \text { Anti-HBc total } & \text { Anti-HBs } & \text { Anti-HD total } \\ & & \% & \% & \% & \% \\ \text { Apurinã } & \text { Amazonas } & 18,1 & 64,4 & - & 4,9 \\ \text { Caiabi } & \text { Mato Grosso } & 1,9 & 19,8 & - & 0 \\ \text { Cinta Larga } & \text { Rondônia } & 1,0 & - & 6,1 & \text { - } \\ \text { Deni } & \text { Amazonas } & 0 & 48,1 & - & 0 \\ \text { Jamamadi } & \text { Amazonas } & 0 & 19,7 & - & 0 \\ \text { Kayapó } & \text { Pará } & 1,2 & - & 22,1 & \text { - } \\ \text { Kanamari } & \text { Amazonas } & 0 & 78,6 & - & 6,8 \\ \text { Karitiana } & \text { Rondônia } & 3,4 & 35,3 & 16,1 & \text { - } \\ \text { Kulina } & \text { Amazonas } & 11,9 & 67,7 & - & 7,7 \\ \text { Mundurucú } & \text { Pará } & 0,6 & - & 22,0 & - \\ \text { Mura-Pirahã } & \text { Amazonas } & 0 & 32,3 & - & 0 \\ \text { Parakanã } & \text { Pará } & 14,4 & 84,7 & - & 0 \\ \text { Paumari } & \text { Amazonas } & 20,6 & 62,0 & - & 4,2 \\ \text { Suruí } & \text { Rondônia } & 11,3 & - & 24,2 & \text { - } \\ \text { Tikuna } & \text { Amazonas } & 0,8 & 64,4 & - & \text { - } \\ \text { Txucarramãe } & \text { Mato Grosso } & 6,9 & 66,4 & - & 0 \\ \text { Urubu-Kaapor } & \text { Maranhão } & 1,5 & - & 6,4 & \text { - } \\ \text { Wayana-Apalai } & \text { Pará } & 14,2 & - & 26,3 & - \\ \text { Yanomami } & \text { Roraima } & 7,5 & - & 12,3 & \text { - } \\ \text { Yanomani } & \text { Amazonas } & 11,3 & 95,7 & 78,7 & 0 \\ & & & & & \end{array}$

Tabela 2 - Prevalência do AgHBs entre indígenas da Amazônia Brasileira por grupo de idade e etnia.

$\begin{array}{lrrrrrr}\text { Faixa etária } & \text { Txucarramãe \% } & \text { Caiabi \% } & \text { Karitiana \% } & \text { Apurinã \% } & \text { Kulina \% } & \text { Paumari \% } \\ 0-10 & 9,7 & 2,9 & 0 & 22,4 & 5,9 & 21,7 \\ 10-20 & 15,6 & 3,6 & 5,4 & 7,1 & 13,8 & 18,4 \\ 20-30 & - & - & 3,0 & 16,7 & 16,0 & 14,8 \\ 30-40 & - & - & 5,5 & 50,0 & 14,3 & 15,4 \\ 40-50 & - & - & - & 12,5 & 33,3 & 33,3 \\ 50-60 & - & - & - & 12,5 & 0 & 66,7 \\ >60 & - & - & - & 0 & 16,7 & 33,3 \\ \text { Total } & 6,9 & 1,9 & 3,4 & 18,1 & 11,9 & 20,6\end{array}$

Yanomami no Amazonas. Taxas relativamente baixas também são encontradas, como entre os Jamamadi no Amazonas, e Caiabi no Mato Grosso, de pouco mais de 19\% ( Tabela 1). É relatado também, para a presença deste marcador, variação significativa entre as aldeias visitadas, inclusive da mesma etnia ${ }^{8}$. Aprevalência do anti-HBc total apresenta um gradiente crescente, em relação a idade, chegando a níveis bastante elevados, cerca de $100 \%$ em indivíduos acima dos 80 anos (Tabela 3).

Tabela 3 - Prevalência deinfecção passada pelo VHB (anti-HBc total reativos) entre indígenas da Amazônia brasileira por grupo de idade e etnia.

\begin{tabular}{lcrrrrrr} 
Faixa etária & Txucarramãe & Caiabi & Karitiana & Apurinã & \multicolumn{2}{c}{ Kulina } & Paumari \\
& $\%$ & \multicolumn{1}{c}{$\%$} & \multicolumn{1}{c}{$\%$} & \multicolumn{1}{c}{$\%$} & \multicolumn{1}{c}{$\%$} & \multicolumn{1}{c}{$\%$} & \multicolumn{1}{c}{$\%$} \\
\hline $0-10$ & 73,8 & 6,3 & 6,2 & 34,2 & 12,5 & 8,3 & 3,4 \\
$10-20$ & 91,0 & 60,7 & 27,0 & 76,9 & 60,0 & 83,9 & 10,0 \\
$20-30$ & - & - & 36,4 & 80,0 & 85,2 & 91,3 & 25,0 \\
$30-40$ & - & - & 100,0 & 71,4 & 94,4 & 90,9 & 57,1 \\
$40-50$ & - & - & - & 71,4 & 100,0 & 100,0 & 0 \\
$50-60$ & - & - & - & 100 & 60,0 & 100,0 & 0 \\
$>60$ & - & - & - & 75,0 & 60,0 & 100,0 & 80,0
\end{tabular}

A prevalência de infecção resolvida, indivíduos anti-HBs reativos, varia em torno dos $20 \%$, principalmente naquelas etnias que apresentam padrão de elevada endemicidade.

A presença do anti-HD total só é relatada em grupos da Amazônia ocidental, no Estado do Amazonas, a prevalência variando de $0 \%$ nos grupos que apresentaram baixa taxas de infecção do VHB, a 7,7\% entre os Kulina, grupo com elevada taxa de portador do AgHBs. Nos grupos da Amazônia oriental, Pará, Rondônia, Mato Grosso e Roraima, não foi encontrado marcadores sorológicos do VHD ( Tabela 1). Esteve presente em todas as faixas de idade, entretanto, a prevalência parecer ser mais marcante nos indivíduos entre quinze e quarenta anos ( Tabela 4).

Tabela 4 - Prevalência de infecção pelo VHD (anti-HD total reativos) entre indígenas da Amazônia brasileira por grupo de idade e etnia.

\begin{tabular}{lcrc}
\hline Faixa etária & Apurinã $\%$ & Kulina \% & Paumari \% \\
\hline $0-10$ & 4,2 & 0 & 7,7 \\
$10-20$ & 6,1 & 21,1 & 0 \\
$20-30$ & 6,7 & 3,8 & 0 \\
$30-40$ & 8,3 & 5,0 & 8,3 \\
$40-50$ & 0 & 0 & 33,3 \\
$50-60$ & 0 & 0 & 0 \\
$>60$ & 0 & 0 & 33,3 \\
Total & 4,9 & 7,7 & 4,2 \\
\hline
\end{tabular}




\section{PROVÁVEIS MECANISMOS DE TRANSMISSÃO}

A natureza da maioria das publicações não permite estabelecer relação de causalidade, basicamente por avaliarem amostras de conveniência. Algumas investigações, entretanto, obedecem certos rigores metodológicos mesmo considerando a dificuldade operacional de obedecer protocolos de pesquisa na Amazônia, especialmente com populações indígenas.

Sabemos, no entanto, que a transmissão se dá muito precoce, principalmente entre crianças de cinco a doze anos e entre adultos jovens, que se contaminam provavelmente por transmissão horizontal familiar e por contato sexual, entre os jovens ${ }^{281338}$. A transmissão familiar, se mostra muito importante, inclusive demonstrado em estudos de biologia molecular, enquanto a transmissão vertical, deve contribuir muito pouco para a disseminação destes vírus nesta populaçãa $0^{917} 33$.

Fatores socioculturais como: densidade populacional, 0 costume de realizar escarificações, tatuagens, atividade sexual, e 0 hábito de processar alimentos oralmente, são implicados como importantes na transmissã $0^{515}$.

Fatores da constituição genética da população amazônica, são citados como prováveis mediadores importantes na modulação da resposta imunológica, favorecendo a manutenção do estado de portador do $\mathrm{AgHBs}$ na região ${ }^{36}$.

A ecologia da região, a interação peculiar do homem amazônico com o meio ambiente, a possibilidade de transmissão por vetores e a formação histórica desses povos em relação a forma e tempo de contato com a civilização brasileira, são descritos também como fatores determinantes deste padrão peculiar de distribuição da infecção pelo VHB e VHD ${ }^{811} 25343536$.

\section{DISCUSSÃO}

Embora a representatividade de grande parte dos estudos avaliados possa ser questionada, e na maioria das vezes as análises sejam feitas com bases em amostras de conveniência, ou de estudos epidemiológicos de surtos de hepatite fulminante, fica evidente a importância tanto do espectro alargado da transmissão do VHB e VHD, como do impacto na morbidade e mortalidade de populações indígenas da Amazônia2 813151720222931323839 .

Os dados avaliados revelam padrão heterogêneo de distribuição do VHB entre as etnias já estudadas, padrão descrito, inclusive em um mesmo grupo étnico, entre aldeias muito próximas geograficamente ${ }^{8,38}$. Este padrão não é peculiaridade da Amazônia brasileira, é descrito também na Amazônia de outros países da América do sul1011 122226293139 , e em outras regiões de elevada endemicidade como no Alasca ${ }^{27}$, certas regiões da África, sul da Ásia e ilhas do Pacífico ${ }^{28} 3040$.

A transmissão em idades precoces orienta para a implantação ou avaliação rigorosa das medidas de controle, estudos relatam somente imunogenicidade de vacinas ${ }^{16}$, sem referências ao impacto da vacinação nas taxas de prevalência de marcadores do VHB e VHD em comunidades indígenas. A transmissão horizontal familiar por mecanismos ainda obscuros, junto com a origem dessas viroses no continente sul americano são os grandes desafios ainda a serem revelados.

Interações complexas particulares da região, entre aspectos do ambiente, agente e do hospedeiro, podem ser determinantes do padrão heterogêneo, da gravidade e da manutenção da transmissão.

A epidemiologia da infecção pelo VHB e VHD em comunidades indígenas talvez guarde e seja capaz de revelar aspectos importantes para a compreensão da origem dessas viroses na região. Sabemos que em toda a Amazônia prevalece 0 genótipo $\mathrm{F}$ do VHB, tido como característico da população da

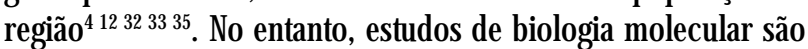
raros. Acreditamos que a implantação de ações de vigilância epidemiológica e estudos com bases populacional, com protocolos específicos para populações indígenas, aliando técnicas de biologia molecular a estudos antropológicos, sejam decisivas para 0 avanço do conhecimento dessas questões.

0 VHD é descrito somente entre grupos da região ocidental do Estado do Amazonas, associado a taxas elevadas de portadores do AgHBs, prevalecendo entre adultos jovens, provavelmente se disseminando, principalmente, por contato sexual ${ }^{8}$. Apesar de estar ausente na Amazônia oriental, Roraima, Mato Grosso e Rondônia, a presença marcada do VHB, e como o processo de integração implica em grandes migrações, estes grupos devem ser considerados em risco potencial de contaminação.

A influência marcante do VHD na morbidade e mortalidade desta população, é facilitada pela intensa circulação do VHB em idades precoces, com conseqüente risco elevado de desenvolvimento de estado de portador crônico, estando o VHD fortemente associado a surtos familiares de hepatite fulminante e casos de doenças crônicas do fígado em indivíduos com menos de vinte anos de idade ${ }^{18}$.

Considerando que o VHD apresenta características biológicas semelhantes aos virióides de plantas, e a complexidade e biodiversidade do ecossistema amazônico, foi sugerido que estes fatores somados a fatores do hospedeiro, pudessem influenciar neste padrão peculiar de severidade que assume a infecção pelo VHD na região $0^{1011} 12182229313639$.

Estudo de casos de hepatite aguda entre militares da Amazônia Peruana, 95\% com sorologia indicativa de infecção aguda pelo VHB, sendo $65 \%$ destes coinfectados pelo VHD, demonstrou a relação entre a severidade dos casos e a associação do genótico III do VHD com genótipo F do VHB, descritos como cepas originárias do Novo Mundo ${ }^{12}$.

Entre grupos indígenas da Venezuela, esta questão não fica tão evidente, pois apesar de confirmar a predominância do genótipo $\mathrm{F}$ do VHB, mostra a associação deste com 0 genótipo I do VHD, comum nos países da Europa, sugerindo que este tenha sido introduzido no processo de colonização $0^{35}$.

Após 0 uso em programas de vacinação de massa, por mais de dez anos, da vacina contra hepatite $B$, se discute as significativas quedas nas taxas de prevalência de marcadores sorológicos do VHB e VHD. Os dados são realmente 
animadores, e publicações chegam a falar em erradicação dessas viroses ${ }^{21}$, alguns países relatam taxas de portadores do AgHBs até cinco vezes menor, que a anterior a introdução da vacina ${ }^{14} 2324$.

Avanços devem ser alcançados no conhecimento da origem do VHB e VHD na Amazônia. As populações indígenas com sua memória genética são, na verdade, 0 experimento ao vivo, 0 que demanda um inquérito abrangente, avaliando a influência dos diversos aspectos históricos, ecológicos, médicos e antropológicos envolvidos.

\section{REFERÊNCIAS BIBLIOGRÁFICAS}

1. Alecrim WD, Marreiros LS, Alecrim MGC, Miranda Santos IKF. Inquérito sobre presença de HBsAg em habitantes da Lábrea-Amazonas. Revista da Sociedade Brasileira de Medicina Tropical 19: 58-59, 1986.

2. Azevedo RA, Silva AE, Ferraz MLG, Marcopito LF, Baruzzi RG. Prevalência dos marcadores sorológicos dos vírus da hepatite B e D em crianças das tribos Caiabi e Txucarramãe do Parque Indígena do Xingu, Brasil, Central. Revista da Sociedade Brasileira de Medicina Tropical 29: 431-439, 1996.

3. Bensabath G, Hadler SC, Soares MPC, Fields H, Maynard JE. Epidemiologic and serologic studies of acute viral hepatitis in Brazil's Amazon Basin. Bulletin of the PanAmerican Health Organization 21: 16-27, 1987.

4. Blitz L, Pujol FH, Swenson PD, Porto L, Atencio R, Araujo M, Costa L, Monsalve DC, Torres JR, Fields HA, Lambert S, Van Geyt C, Norder H, Magnius LO, Echevarria JM, Stuyver L. Antigenic diversity of hepatitis B virus strains of genotype $\mathrm{F}$ in Amerindians and other population groups from Venezuela. Journal Clinical Microbiology 36: 648-51, 1998.

5. Brabin L, Brabin BJ. Cultural factors and transmission of hepatitis B virus. American Journal of Epidemiology 122: 725-730, 1985.

6. Braga WSM. Relatório de Investigação de suspeita de ocorrência de casos de hepatites virais entre populações indígenas do vale do rio Javari. Investigação realizada através da Coordenadoria de Vigilância à Saúde da Superintendência de Saúde do Estado do Amazonas, 1995.

7. Braga WSM, Brasil LM, Panula M, Carvalho JAB. Epidemiological aspects of hepatitis B virus (HBV) infection in children, Beruri, Amazonas. Acta Hepatologica 1: 23, 1991.

8. Braga WSM, Brasil LM, Souza RAB, Castilho MC, Fonseca JCF. Ocorrência da infecção pelo vírus da hepatite B (VHB) e delta (VHD) em sete grupos indígenas do Estado do Amazonas. Revista da Sociedade Brasileira de Medicina Tropical 34: 349-355, 2001.

9. Brasil LM, Braga WSM, Souza RAB, Castilho MC, Fonseca JCE. The prevalence of hepatitis B virus (HBV) markers within household in the state of Amazonas, Brazil. Hepatology 19: 451I, 1994.

10. Buitrago B, Popper H, Hadler SC, Thung SN, Gerber MA, Purcell RH, Maynard JE. Specific histologic features of Santa Marta Hepatitis: a severe form of hepatitis delta-virus infection in Northern South America. Hepatology 6: 1285-1291, 1986.

11. Cabezas C, Ramos F, Sanches J, Cobos M, Watts D, Callanan J, Gotuzzo E. High prevalence of infection with hepatitis B and Delta in the children of Huanta - Ayacucho (Peru). In: Abstract of IX Triennial International Symposium on Viral Hepatitis and Liver Disease, Italy p. 118, 1996.

12. Casey JL, Niro GA, Engle RE, Vega A, Gomez H, McCarthy M, Watts DM, Hyams KC, Gerin JL. Hepatitis B virus ( HBV)/hepatitis D virus (HDV) coinfection in outbreaks of acute hepatitis in the Peruvian Amazon basin: the roles of HDV genotype III and HBV genotype F. Journal of Infectious Diseases 174: 920-6, 1996.

13. Castro EJ, Rosa Filho SM. Prevalência dos marcadores dos vírus B e Delta em população indígena da Tribo Yanomami (AM) . Moderna Hepatologia 1: 21,1989 .

14. Chen DS, Hsu HM, Chang MH, SungJL, and the Hepatitis Control Committee. Hepatitis B vaccine status report on long-term efficacy, In: Rizzetto M,
Purcell RH, Gerin JL, Verme G (eds) Viral Hepatitis and Liver Diseases, Italy, p.635-637, 1997.

15. Coimbra CEA, Santos RV, Yoshida CFY, Baptista ML, Flowers NM, Valle ACF. Hepatitis B epidemiology and cultural practices in amerindian populations of Amazonia: The Tupí-Mondé and Xavante from Brasil. Society Science Medicine 42: 1735-43, 1996.

16. Colichon A, Vildosola H, Sjogren M, Cantella R, Safary A. The serological response to a recombinant DNA vaccine in the native residents of two hyperendemic hepatitis B areas in the Peruvian Amazonia. Revista de Gastroenterologia del Peru 13: 78-84, 1993.

17. Ferrari J0, Ferreira MU, Tanaka A, Mizokami M. The seroprevalence of hepatitis B and Cin an Amerindian population in the southwestern Brazilian Amazon. Revista da Sociedade Brasileira de Medicina Tropical 32: 299302, 1999.

18. Fonseca JCF, Brasil LM, Castilho MC, Braga WSM, Souza RAB, Ferreira LCL. Hepatitis delta virus ( HDV) infectious in the Brazilian Amazon basin and its role in chronic liver disease. Hepatology 19: 63I, 1994.

19. Fonseca JCF, Brasil LM, Castilho MC, Souza RAB, Braga WSM. The occurence of increased rates of HAV, HBV and HDV infection in an isolated village, Ipixuna, Amazonas, Brazil. Hepatology 19: 63I, 1994.

20. Fonseca JCF, Castejon MJ, Cesario ALO, Baroni M. Prevalência da infecção pelos vírus das hepatites $\mathrm{B}$ e delta em indígenas da nação Tikunas, alto rio Solimões, Amazonas, Brasil. In: Resumos do X Congresso Brasileiro de Hepatologia p. E-084, 1988.

21. Gaeta GB, Stroffolini T, Chiaramonte M, Ascione T, Stornaiuolo G, Lobello S, Sagnelli E, Brunetto MR, Rizzetto M. Chronic hepatitis D: a vanishing Disease? An Italian multicenter study. Hepatology 32: 824-827, 2000.

22. Hadler SC, Monzon M, Ponzetto A, Anzola E, Rivero D, Mondolfi A, Bracho A, Francis DP, Geber MA, Thung S, Gerin J, Maynard JE, Popper H, Purcell RH. Delta virus infection and severe hepatitis: An epidemic in Yucpa Indians of Venezuela. Annals of Internal Medicine 100: 339-344, 1984.

23. Hall AJ, Inskip HM, Loik F, Chotard J, Jawara M, Vall Mayans M, Greenwood BM, Whittlel H, Njie ABH, Cham K, Bosch X, Muir CS. Hepatitis B vaccine in the Expanded Program on Immunization: The Gambian experience. Lancet I: 1057-1059, 1989.

24. Harpaz R, MacMahon BJ, Margolis HS, Shapiro CN, Havron D, Carpenter G, Bulkow LR, Wainwright RB. Elimination of New Chronic Hepatitis B Virus Infections: Results of the Alaska Immunization Program. The Journal of Infectious Diseases 181: 413-418, 2000.

25. Hyams KC. Mosquito transmission of hepatitis B. Tropical Geographic Medicine 41: 185-189, 1989.

26. Leon P, Venegas E, Bengoechea L, Rojas E, Lopez JA, Elola C, Echevarria JM. Prevalence of infections by hepatitis B, C, D and E viruses in Bolivia. Revista Panamericana de Salud Publica 5: 144-151, 1999.

27. MacMahon BJ, Schoenberg S, Bulkow L,Wainwright RB, Fitzgerald MA, Parkinson AJ, Coker E, Ritter D. Seroprevalence of hepatitis B viral markers in 52,000 Alaska natives. American Journal of Epidemiology 138: 544-549, 1993.

28. Maher CP, Harris MS, Milne A, Johnston A, Stewart A, Waldon JA. Seroepidemiology of hepatitis B infection in children in Vanatu. Medicine Journal Austry 154: 249-253, 1991.

29. Manock SR, Kelley PM, Hyams KC, Douce R, Smalligan RD, Watts DM, Sharp TW, Casey JL, Gerin JL, Engle R, Alava-Alprecht A, Martinez CM, Bravo NB, Guevara AG, Russel KL, Mendoza W, Vimos C. An outbreak of fulminant hepatitis delta in the Woarani, an indigenous people of the Amazon basin of Ecuador. American Journal of Tropical Medicine and Hygiene 63: 209-213, 2000.

30. Maynard JE. Hepatitis B global importance and need for control. Vaccine 8: 518-520, 1990.

31. Mendez M, Arce M, Kruger HP, Sanches SA. Prevalência de marcadores serologicos de hepatitis virica en diversos grupos de poblacion del Peru. Bulletin of the PanAmerican Health Organization 106: 127-138, 1989.

32. Nakano T, Lu L, Hu X, Mizokami M, Orito E, Shapiro C, Hadler S, Robertson B. Characterization of hepatitis B virus genotypes among Yucpa Indians in Venezuela. Journal Genetic Virology 82: 359-365, 2001. 
33. Niro GA, Casey JL, Gravinese E, Garrubba M, Conoscitore P, Sagnelli E, Durazzo M, Caporaso N, Perri F, Leandro G, Facciorusso D, Rizzetto M, Andriulli A. Intrafamilial transmission of hepatitis delta virus: molecular evidence. Journal Hepatology 30: 564-569, 1999.

34. Paula VS, Arruda ME, Vitral CL, Gaspar AM. Seroprevalence of viral hepatitis in riverine communities from the Western Region of the Brazilian Amazon Basin. Memórias do Instituto Oswaldo Cruz 96: 1123-1128, 2001.

35. Quintero A, Uzcategui N, Loureiro CL, Villegas L, Illarramendi X, Guevara ME, Ludert JE, Blitz L, Liprandi F, Pujol FH. Hepatitis delta virus genotype I and III circulate associated with hepatitis B virus genotype F in Venezuela. Journal Medical Virology 64: 356-359, 2001

36. Santos AK, Ishak MO, Santos SE, Guerreiro JF, Ishak R. A possible correlation between the host genetic background in the epidemiology of hepatitis B virus in the Amazon region of Brazil. Memórias do Instituto Oswaldo Cruz 90: 435-442, 1995.

37. Soares MCP, Bensabath G. Tribos indígenas da Amazônia oriental como população de risco para a hepatite D (Delta). Revista do Instituto de Medicina Tropical de São Paulo 33: 241, 1991.

38. Soares MCP, Menezes RC, Martins SJ, Bensabath G. Epidemiologia dos virus das hepatitis B, C e D na tribo indígena Parakanã, Amazônia oriental brasileira. Boletin de la Oficina Sanitaria Panamericana 117: 124-135, 1994.

39. Torres JR, Mondolfi A. Protacted outbreak of severe Delta hepatitis: experience in an isolated Amerindian population of the upper Orinoco Basin. Reviews of Infectious Diseases 13: 52-55, 1991.

40. World Health Organization Prevention and control of hepatitis B in the community. Communicable Disease Series 1: 17-26, 1996. 